\title{
Optics Design for Microwave Imaging Reflectometry in LHD
}

\author{
Tomokazu YOSHINAGA, Yoshio NAGAYAMA, Daisuke KUWAHARA ${ }^{1)}$, Hayato TSUCHIYA, \\ Soichiro YAMAGUCHI ${ }^{2)}$, Yuichiro KOGI ${ }^{3)}$, Shunji TSUJI-IIO ${ }^{1)}$, Hitoshi HOJO*,4) \\ and Atsushi MASE ${ }^{5}$ \\ National Institute for Fusion Science, 322-6 Oroshi, Toki 509-5292, Japan \\ ${ }^{1)}$ Tokyo Institute of Technology, 2-12-1 Ookayama, Meguro 152-8550, Japan \\ ${ }^{2)}$ Kansai University, 3-3-35 Yamate, Suita 564-8680, Japan \\ ${ }^{3)}$ Fukuoka Institute of Technology, 3-30-1 Wajiro-Higashi, Fukuoka 811-0295, Japan \\ ${ }^{4)}$ University of Tsukuba, 1-1-1 Tennodai, Tsukuba 305-8577, Japan \\ ${ }^{5)}$ Kyushu University, 6-1 Kasuga-Koen, Kasuga 816-8680, Japan
}

(Received 26 May 2010 / Accepted 1 July 2010)

\begin{abstract}
An optics system for microwave imaging reflectometry (MIR) in the Large Helical Device (LHD) was newly developed to optimize the performance of the two-dimensional microwave receiver array. Reflected microwaves from the plasma and the first local oscillator (LO) wave are transmitted to the receiver array via the optics from the front. Finite-difference time-domain (FDTD) calculation was used to design the ellipsoidal or hyperboloidal shapes of the quasi-optical mirrors. It is confirmed that the LO beam in the constructed system covers the receiver antenna aperture area as intended. The $\mathrm{S} / \mathrm{N}$ ratios of the signals are improved with this optimized optics system from those in the previous system.
\end{abstract}

(c) 2010 The Japan Society of Plasma Science and Nuclear Fusion Research

Keywords: microwave imaging reflectometry, quasi-optics design, finite-difference time-domain method

DOI: $10.1585 /$ pfr.5.030

Understanding confinement and transport in magnetically confined plasmas is one of the important issues for realizing fusion reactor. Microscale instabilities such as turbulence are considered to activate anomalous transport. Microwave imaging reflectometry (MIR) would be a sensitive fluctuation diagnostics in turbulent plasmas. MIR is a multi-receiver reflectometry that can potentially visualize the 3-D structures of electron density fluctuations by projecting images of the cutoff surfaces onto the image focal plane [1].

In the Large Helical Device (LHD), a prototype MIR system with three commercial horn antennas, has successfully received scattered microwaves from plasmas [2,3]. The received microwaves were transmitted from the receiver antenna to mixer components remote from the diagnostic port by oversized waveguides. In a full-scale imaging system, however, it is difficult to use waveguides to transmit received signals from dozens of receiver channels. This signal transmission problem was solved by a newly developed 2-D horn-antenna mixer array (HMA), which consists of arrayed quasi-optical antenna-mixers covered with pyramidal horn apertures $[4,5]$. This HMA was developed to down-convert received microwaves into intermediate frequency (IF) signals by Schottky diodes, which are placed inside each of the HMA apertures. After downconversion, the signal handling becomes very convenient,

author's e-mail: yoshinaga.tomokazu@lhd.nifs.ac.jp

* deceased

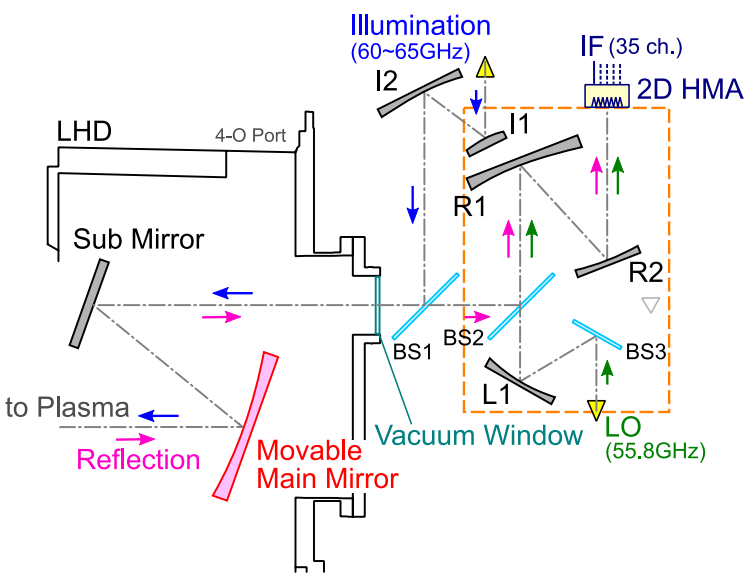

Fig. 1 Schematic view of MIR system in LHD.

since printed circuit boards or ordinary coaxial cables can be used for amplification, filtering, or transmission.

This paper presents the methodology for mixing the received microwave signal with the first local oscillator (LO) signal for down-conversion. Our technique is to cast the LO microwave $(55.8 \mathrm{GHz})$ from the front of the HMA aperture together with the received microwave signals. The status of the optics system for LO wave projection is also described.

The newly designed optics system for MIR in LHD is illustrated in Fig. 1. It consists of aluminum alloy mirrors 


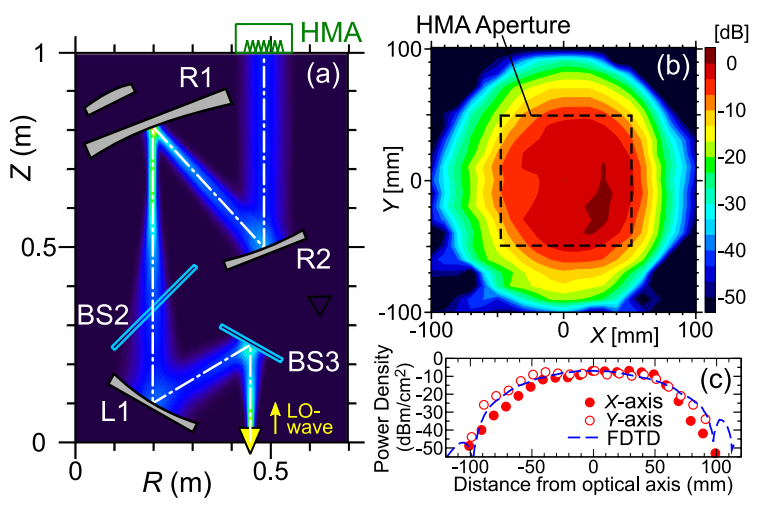

Fig. 2 (a) LO beam intensity calculated by the FDTD method. (b) Normalized power profile of the LO beam on the HMA aperture surface. (c) Power density profile of the LO beam along the $X$-axis (filled circles) and the $Y$-axis (open circles). Dashed line denotes the FDTD-simulated profile along the $X$-axis shown in (a).

and dielectric (acrylic) beam splitters to realize the following three functions. 1) The illuminating probe microwaves in the V-band (55-65 GHz) are projected onto the plasma. 2) The scattered waves from the plasmas are focused on the HMA apertures and received with 35 receiver channels ( 7 in the poloidal direction by 5 in the toroidal direction). 3) The first LO wave is also cast onto the HMA apertures from the front. The down-converted first IF signals from the HMA are down-converted again into the second IF signals at $110 \mathrm{MHz}$ by mixing with the second LO signals and are demodulated/detected to derive the quadrature phase signals and the amplitude signals.

The ellipsoidal or hyperboloidal surfaces of the curved aluminum alloy mirrors were determined for simultaneous focusing of the above three wave components (the illuminating waves, the scattered waves, and the first LO wave). The focusing optics of the scattered waves on the HMA aperture (R1 and R2) and the illuminating optics for probe waves (I1 and I2) were designed first to match the existing movable main mirror. The LO wave transmission optics (L1) was matched to the previously designed R1 so that the LO beam covers the whole HMA aperture area with a $100 \mathrm{~mm} \times 100 \mathrm{~mm}$ rectangle to supply enough power for down-conversion $(-16 \mathrm{dBm})$ to the mixer diodes in the HMA [5]. Figure 2 (a) shows the designed power density of the beamline of the LO wave, which is numerically calculated by using the 2-D finite-difference time-domain (FDTD) method [6,7]. The computed area is denoted by the rectangle shown in Fig. 1. The coordinates $R$ and $Z$ correspond to the radial and vertical position, respectively. The origin of the coordinates is the lower-left corner of the computed area. Since the numerical solutions of the Maxwell equations are directly computed in the FDTD calculation, the effect of finite wavelength can be intrinsically simulated. Note that the effect of the acrylic beam splitter BS2 is neglected, and the acrylic beam combining plate
BS3 is assumed to be a totally reflecting plate for convenience of calculation. Although these dielectric plates split the LO beam, the position and width of the LO beamline is not affected by these thin (less than $1 \mathrm{~mm}$ ) dielectric plates with a low refractive index $(\sim 1.49)$. Since no strong interference effect arises in these plates in the frequency range of the present MIR system, their reflectivity and transmissivity do not vary greatly. The beam reflected by BS2 and that transmitted through BS3 are damped by microwaveabsorbent materials (ECCOSORB) in the real system. The LO beam is attenuated to $10-13 \%(-10$ to $-8 \mathrm{~dB})$ of the original power level at the HMA aperture plane by these plates.

Figure 2 (b) shows the normalized power density profile of the LO wave beam measured in the constructed optics system on the HMA aperture plane with the total output power from the corrugated LO horn at $+24 \mathrm{dBm}$. The $X$ direction corresponds to the alignment direction of the poloidal channel of the HMA, and the $Y$ direction corresponds to the toroidal channel. It is confirmed that the LO beam with a circular cross section roughly covers the HMA aperture (denoted by dashed-line rectangle), as intended. The power density in the HMA aperture area is within $10 \mathrm{~dB}$ below that at the optical axis except the upper-left and the lower-left corner channels, which are $\sim 15 \mathrm{~dB}$ lower. Figure 2 (c) shows the cross sections of the power density profile of the LO beam along the $X$ - and $Y$-axes. The LO beam has flat profiles, especially inside the HMA aperture area of $-50 \mathrm{~mm} \lesssim X, Y \lesssim 50 \mathrm{~mm}$. The profile along the $Y$-axis agrees better with that predicted by the FDTD simulation than that along the $X$-axis. The power density is roughly $-7 \mathrm{dBm} / \mathrm{cm}^{2}$ at the optical axis, and thus it is higher than $-17 \mathrm{dBm} / \mathrm{cm}^{2}$ in most of the HMA aperture area. By considering the effective aperture area of the HMA receiver $\left(1.2 \mathrm{~cm}^{2}\right)$, the LO power supplied to each HMA channel becomes $\sim-6$ to $-16 \mathrm{dBm}$, which is sufficient to drive the mixer diodes of HMA [5]. The integrated LO power on the HMA aperture plane becomes roughly $+12 \mathrm{dBm}$, which is more than $2 \mathrm{~dB}$ lower $(\sim 40 \%)$ than the value estimated from the LO horn output at $+24 \mathrm{dBm}$ with the attenuation from the dielectric plates at -10 to $-8 \mathrm{~dB}$. The power loss would be due to the effects of the side lobe of the LO horn, stray beams from the finite-sized mirrors, or misalignments.

Figure 3 compares an example of the MIR amplitude signal obtained after the optics optimization (shot 96253) (Fig. 3(a)) with the signal before optimization (shot 89643) (Fig. 3 (b)). The signals from the center channel of the HMA during a high ion-temperature operation are plotted. The working frequency is $\sim 62 \mathrm{GHz}$ in shot 96253 and $\sim 56 \mathrm{GHz}$ in shot 89643 . The signal level from the plasma has increased since the replacement of the optics system. Here, the durations of the plasma discharges are considered as periods during which a finite plasma density can be observed. An increase in the plasma signal has been observed among most of the HMA channels, including the 

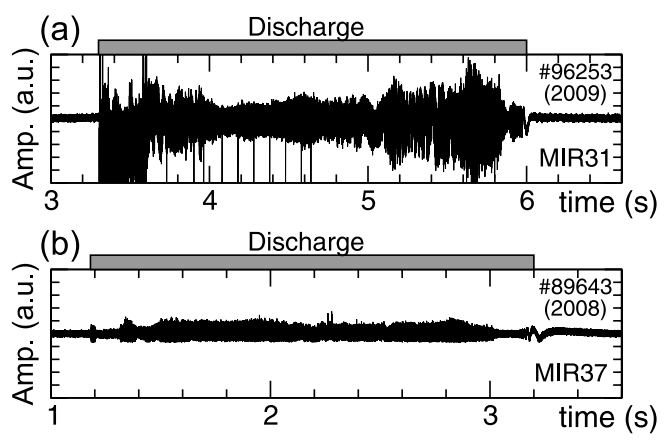

Fig. 3 Typical waveforms of MIR signals in high ion temperature operation after (a) and before (b) optimization of the optics system.

edge channels whose LO inputs are relatively low. The noise level, which is defined as the signal level before and after the discharge pulse, would depend on the stability of the oscillators and amplifiers in the signal generator section for the probe waves and the LO wave. The negative spikes found from 3.7 to $4.7 \mathrm{~s}$ in shot 96253 (Fig. 3 (a)) are synchronized with the switching off of the neutral beam pulses. Further interpretation of the phenomena is beyond the scope of this paper. Although the probing frequencies and powers as well as the plasma conditions differ, their effects on the $\mathrm{S} / \mathrm{N}$ ratios are not large in this comparison. Thus, the improvement of the $\mathrm{S} / \mathrm{N}$ ratio from shot 89643 (Fig. 3 (b)) to shot 96253 (Fig. 3 (a)) can be attributed to the modification of the optics system.

In conclusion, the optics system for MIR diagnostics in LHD was redesigned using FDTD simulation and was constructed. The obtained power profile of the LO beam agrees roughly with the predicted profile. The $\mathrm{S} / \mathrm{N}$ ratios of the MIR signals are improved with this new optics system. This study was supported by the NINS Imaging Science Project (NIFS09KEIN0021), by the Grant-inAid for Scientific Research (21246140) and by the NIFS (NIFS09ULPP525).

[1] H. Park et al., Rev. Sci. Instrum. 74, 4239 (2003).

[2] S. Yamaguchi et al., Rev. Sci. Instrum. 77, 10E930 (2006).

[3] S. Yamaguchi et al., Plasma Fusion Res. 2, S1038 (2007).

[4] D. Kuwahara et al., J. Plasma Fusion Res. SERIES 8, 649 (2009).

[5] D. Kuwahara et al., J. Plasma Fusion Res SERIES 9, 125 (2010).

[6] M. Ignatenko et al., Nucl. Fusion 46, S760 (2006).

[7] H. Hojo et al., Rev. Sci. Instrum. 70, 983 (1999). 